\title{
Pedestrians' crossing behaviour in south-western Nigeria
}

\author{
Moses Olaniran Olawole*, Adewale Mukhtar Olayiwola
}

Obafemi Awolowo University, Nigeria

This study examined the pedestrians' safe and unsafe behaviour before and during street crossing in three cities in South Western Nigeria. 1,214 pedestrians were observed at crossing sites using direct observation approach. The data were analysed using descriptive statistics, chi-square, Analysis of Variance (ANOVA) and logistic regression. In all, 730 $(60.1 \%)$ of the observed pedestrians were male, and 484 (39.9\%) were female. The analysis of the data showed that safe crossing practices were generally high, as over $50 \%$ of pedestrians at different stages of the crossing process were categorized as exhibiting safe observation behaviour. In terms of crossing tempo, Chi-square test revealed significant difference between male $(65.1 \%)$ than female $(56.0 \%)$ pedestrians $\left(\chi^{2}=10.12, d=1, p=0.000\right)$. While ANOVA showed significant variation in the risky crossing behaviour among the cities $(\mathrm{F}(2$, $1211)=71.93, \mathrm{P}<0.01)$. Logistic regression analysis showed that adult pedestrians are 0.79 times more likely to exhibit safe crossing behaviour than younger pedestrians and pedestrians are 0.42 times more likely to exhibit safe crossing when vehicles are moving on one side of the road than when there is no vehicular movement. The study suggests measures to enhance safe street crossing behaviour in the sampled cities and other cities in Nigeria..

Key Words: pedestrians, street, crossing behaviour, safety, head movements, Nigeria.

Article Info: Received: May 25, 2018; Revised: November 7, 2018; Accepted: November 15, 2018; Online: November 30, 2018.

\section{Introduction}

Road traffic injuries claimed more than 1.2 million lives each year. Majority of the fatality occurred in low and middle-income countries. These group of countries also accounted for about twice the fatality rates registered in highincome countries and 90\% of global road traffic deaths (WHO, 2015).

\section{* Corresponding author}

Address: Department of Geography, Obafemi Awolowo University, Ile-Ife, Osun, 220282, Nigeria.

Phone: +2348035784825 | Email: molawole@oauife.edu.ng

(C)2018 Human Geographies; The authors

(c) (7) This work is licensed under a

Creative Commons Attribution 4.0 International License. DOI:10.5719/hgeo.2018.122.5 
Pedestrian injuries and fatalities make up half of these fatalities. In Africa, pedestrians account for 30\% of road traffic deaths (WHO, 2015). The proportion of the pedestrians involved in road traffic crashes vary from one country to the other. A study in Ghana found that $68 \%$ of the pedestrian killed were knocked down by a vehicle when they were in the middle of the roadway (Damsere-Derry et al., 2010). In another study, Ogendi et al. (2013) reported that out of the 176 persons involved in road traffic crashes in Kenya and admitted in Kenya National Hospital (KNH) in 2011, the highest proportion $(59.1 \%)$ of road traffic injury were comprised of pedestrians. The study also revealed that $72.6 \%$ of the pedestrians involved were injured while crossing the road, $11 \%$ were standing by the road while $8.2 \%$ were walking along the road, and another $8.2 \%$ were hit while engaging in other activities including hawking (Ogendi et al., 2013).

The trend is similar in Nigeria, for instance, Aladelusi et al. (2014) found that $22.9 \%$ of all victims of road traffic crashes examined at the Accident and Emergency Department and the Department of Oral and Maxillofacial Surgery at the University College Hospital, Ibadan between April 2011 and November 2011, were victims of pedestrian road traffic crashes. Also, Solagberu et al. (2015) found that out of 702 pedestrians involved in road crashes, two-thirds of the collisions resulted from road crossing instances.

Based on the rising trend in pedestrians' fatality, issues concerning the behaviour of pedestrians at crossing sites and factors determining pedestrians crossing behaviour have been examined in several studies in developed countries. Most of the findings supported the notion that safe and unsafe crossing behaviour of pedestrians varies between genders and age (Diaz, 2002; Tiwari et al., 2007; Tom and Granié, 2011). For instance, Diaz (2002) examined the intention of two adult age groups, under and over 26 years, to perform illegal mid-block crossing using a questionnaire survey and found that younger people had a more positive attitude towards crossing in such risky situations than the older target group who perceived more acceptances from significant others and had a lower Perceived Behavioural Control (PBC).

The outcomes of many gender studies suggest that individual characteristics are determinants in the decision-making process. For example, studies have shown that male pedestrians expect less negative outcomes of traffic violations than female (Parker, Manstead, Stradling, Reason and Baxter, 1992), and also violate more rules than female pedestrians (Yagil, 2000; Rosenbloom, Nemrodov and Barkan, 2004).

The impact that individual or group actions have on crossing behaviour formed the focus of other studies. For example, Rosenbloom (2009) in the study of individual and group behaviour at red light established that pedestrians were more prone to crossing the road at the red light if they were alone at the pedestrian crossing than pedestrians waiting as a group for the green light. Pesić et al. (2016) found that the presence of accompanying pedestrians increased the risky behaviour of pedestrians and that older pedestrians behaved more safely in traffic while crossing the street.

Studies have also shown that distractions such as wearing headphones, talking on mobile phones, texting etc., influence pedestrians' behaviour (Bungum et al., 2005; Nasar et al., 2008, Pesić et al., 2016). In a recent study, 
Pesić et al. (2016) detail how the use of mobile phones (talking, texting, and listening to music) affects the behaviour of pedestrians during street crossing, showed that pedestrians who used mobile phones while crossing streets behaved less safely than the pedestrians who did not use mobile phones during street crossing. Pedestrian attitudes, perceptions, and behaviour have also formed the focus of several other studies (Sisiopiku and Akin, 2003; Tiwari et al., 2007; Zhou et al., 2009; Huth et al., 2014). Zhuang and Wu (2011) used field observation of 254 pedestrians to study crossing behaviour at unmarked roadways in China. The results showed that $65.7 \%$ of pedestrian did not look for vehicles after arriving at the Kerb. During the crossing, all pedestrians looked at the oncoming vehicles and during interacting with the vehicles, $31.9 \%$ of them ran, and $11.4 \%$ stepped backwards.

None of those mentioned above studies focused on pedestrian behaviour at crossing sites in developing countries. Furthermore, despite the fact that pedestrians' safety behaviours at crossing sites could play a crucial role in the reduction of injuries and fatalities associated with pedestrian crashes, efforts made to understand crossing behaviour and their determinants are scanty in Nigeria and many African countries (Odeleye, 2002; Ogendi et al., 2013; Olawole, 2017). Therefore, this study aims at examining pedestrians crossing behaviour and compliance with safe crossing rules in three medium-sized cities in South Western Nigeria. The objectives are to determine the differences between safe and unsafe crossing behaviour within each of the study cities and between the selected cities. The study also examines the contributions of age, gender, traffic situation and pedestrian crossing as individuals or in groups to safe crossing behaviour of the observed pedestrians in the study cities.

\section{Materials and method}

\section{Study area}

The study area consists of three cities in South Western Nigeria: Ijebu-Igbo (Ogun State), Ile-Ife (Osun State) and Badagry (Lagos State). The three cities that were selected had to respect the following conditions: traditional in nature; medium in population size and located in different states of South Western Nigeria (Figure 1).

Ijebu-Igbo is located in Ijebu North Local Government Area (LGA), Ogun State. It has a land area of $192 \mathrm{~km}^{2}$ and a population of 109,269 (NPC, 2006). The people of Ijebu-Igbo speak the Ijebu dialect, a common dialect among the Ijebus. Most of the people in this city engage in farming and trading. The main cultivated crops are cocoa, cassava, yam and banana. Industrial activities are mostly associated with lumbering. Road transportation accounts for most of the human and freight movement in the city.

Ile-Ife in Osun State is made up of two Local Government Areas; Ife Central and Ife East. It covers an area of about $668,083 \mathrm{~km}^{2}$ and has a population of 644,373 (NPC, 2006). The Ile-Ife community is mostly an old city. The inhabitants of the city engage in farming, commerce and small-scale industrial activities ranging from blacksmithing to lumbering. The transport system in Ile-Ife is 


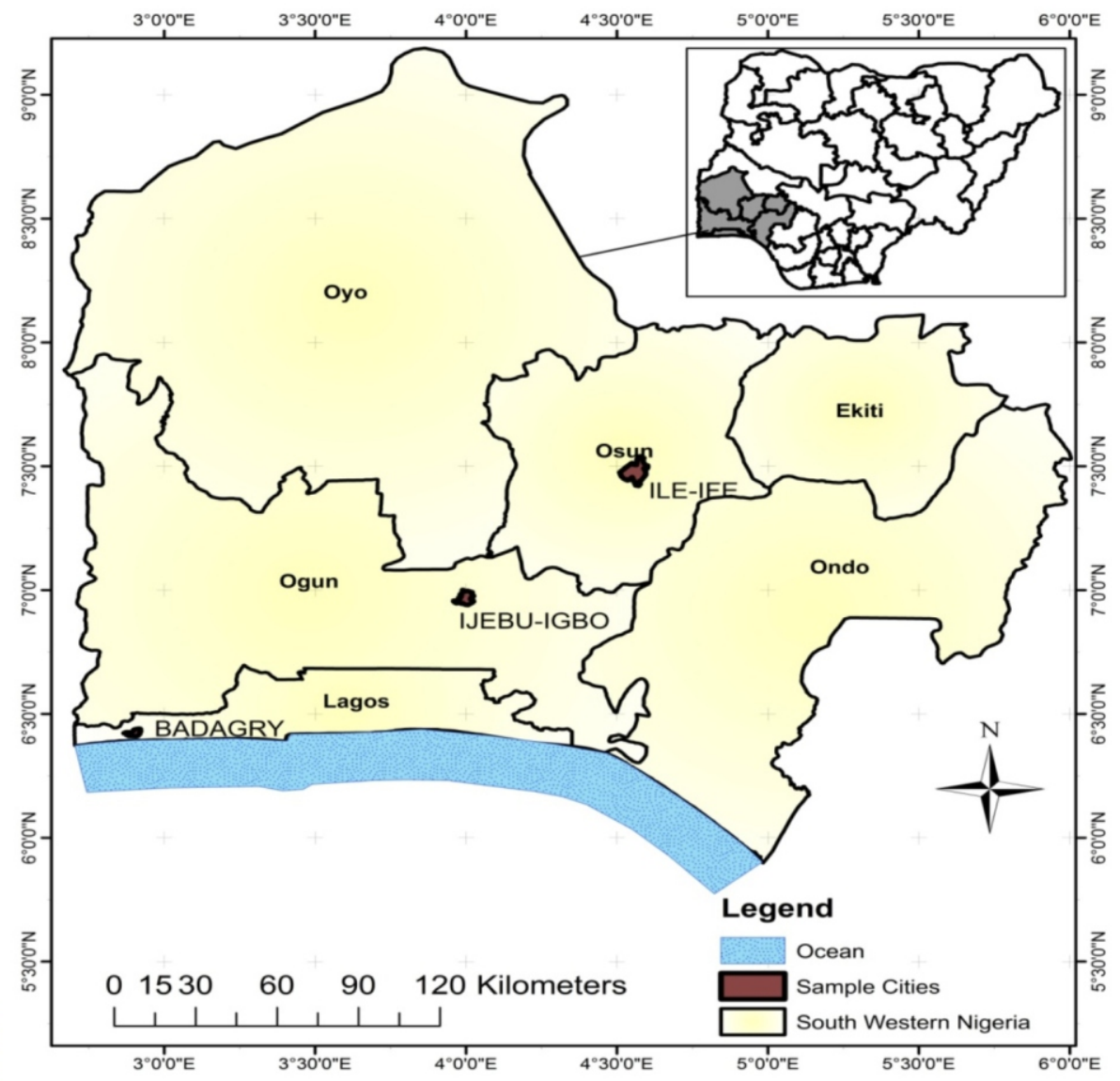

Figure 1. South Western Nigeria Sowing study locations

dominated by road transportation. Transport Infrastructure such as parking lots, traffic light, pedestrian crossing, pedestrian walkway, clearly defined bus stops are lacking in the city (figure 1).

Badagry, is a coastal city and one of the traditional cities in Lagos State, Nigeria. Badagry covers an area of about $441 \mathrm{~km}^{2}$ with an estimated population of 241,093 (NPC, 2006). Given its coastal location, the economy is dominated by fishing activities coupled with trading. The transport system is dominated by road and water transportation. The former consists of a major expressway (Ojo Badagry - Seme expressway) and several other tarred and untarred roads linking the principal towns and settlements together. Among the tarred routes are Badagry - Apa - Owode road, Mowo - Pota - Ikoga road, Agemowo - Ilado Imeke - Ajido road and Badagry - Topo - Iworo - Epe road, which run parallel to the Atlantic coast at the southern part of the city (Olawole, 2008).

Road network represents the principal means for freight and passenger movements in many cities in Nigeria as well as in the selected cities. In the sampled cities, there are three classes of road networks based on ownership: Federal roads (Trunk A or Express Way), State roads (Trunk B or Secondary / Primary Arterial) and Local Government roads (Trunk C or Distributor roads). The Local Government roads form the bulk of the networks within each of the 
Table 1. Road Characteristics and number of crossing sites used in selected cities.

\begin{tabular}{|c|c|c|c|c|c|c|c|c|}
\hline \multirow[t]{2}{*}{ Location } & \multirow{2}{*}{$\begin{array}{l}\text { Road } \\
\text { Type } \\
\text { (Trunk) }\end{array}$} & \multicolumn{3}{|c|}{ Carriage Way } & \multirow{2}{*}{$\begin{array}{l}\text { Central } \\
\text { Divide } \\
\text { (in } \\
\text { metres) }\end{array}$} & \multirow{2}{*}{$\begin{array}{l}\text { Side- } \\
\text { walk } \\
\text { (in } \\
\text { metres) }\end{array}$} & \multirow{2}{*}{$\begin{array}{l}\text { Cro- } \\
\text { ssing } \\
\text { Facility }\end{array}$} & \multirow{2}{*}{$\begin{array}{l}\text { No of } \\
\text { crossing } \\
\text { sites } \\
\text { used }\end{array}$} \\
\hline & & Type & $\begin{array}{l}\text { No of } \\
\text { Lanes }\end{array}$ & $\begin{array}{l}\text { Width of } \\
\text { Lanes (in } \\
\text { metres) }\end{array}$ & & & & \\
\hline Badagry & $\begin{array}{l}\text { Secon- } \\
\text { dary } \\
\text { Arterial } \\
\text { (B) }\end{array}$ & Double & 4 & 3.7 & 1.2 & 2 & Zebra & 4 \\
\hline lle-Ife & $\begin{array}{c}\text { Distri- } \\
\text { butor }(\mathrm{C})\end{array}$ & Single & 2 & 3.5 & - & - & Nil & 4 \\
\hline liebu-Igbo & $\begin{array}{c}\text { Distri- } \\
\text { butor (C) }\end{array}$ & Single & 2 & 3.4 & - & - & Nil & 4 \\
\hline
\end{tabular}

Source: Authors Field Observation, 2017

three cities. These roads are constructed and maintained by the Works Department of Local Government Authorities in each of the cities. This class of roads lacks pedestrian walkways and designated zebra crossing sites. The Federal and State roads are few and not well maintained especially in the selected cities. Pedestrian walkways and crossing sites are available in few of such roads in the study area (figure 1).

\section{Procedure and instrument}

Data for the study were collected based on direct observation of pedestrians' behaviour before reaching the Kerb ( 0.5 to 5 meters), at the Kerb (0 to 0.5 meters) and during street crossing in three medium-sized cities in South Western Nigeria: Ijebu - Igbo (Ogun State), Ile - Ife (Osun State) and Badagry (Lagos State). The technique of data collection adopted involved detail observation and manual recording of pedestrians' behaviour into a pre-designed observation form (grid). The form was designed with some modifications after Tom and Granié (2011) and Poó et al. (2017).

Four streets (one state and three local governments) were purposively selected in each of the three cities. The selected streets were drawn from commercial and residential areas as the sampled cities lack industrial layouts. Characteristics and nature of the roads selected for the study are presented in table 1 and photos $1 \mathrm{~A}$ to $2 \mathrm{D}$. During the survey, observations were made without the pedestrians' knowledge at major crossing sites along the selected streets. Trained observers were stationed strategically, within a distance of 3 to 5 meters of the crossing sites, in shaded areas provided by tressor kiosk, a common feature beside roads in most medium-sized cities in Nigeria.

Each observer randomly selects a pedestrian from either side of the road within a marked distance of 5 meters of the crossing site, observed and recorded his/her behaviours before reaching the Kerb, at the Kerb and during the crossing. The recorded behaviour included: the tempo of pedestrians, the number of pedestrians waiting to cross, head movement(s) and style of crossing before and while crossing. The observed pedestrians were classified into two age categories - youths (15 and 24 years) and adults (25 years and above). In addition, the gender of the observed pedestrian was also recorded. This is in accordance with a similar method used by Rosenbloom (2011) to study traffic light compliance by civilians, soldiers and military officers in Israel. 
Similarly, vehicular movements' during the crossing with respect to three traffic situations: vehicles moving either right or left side; vehicles moving on both sides; and no vehicular movement were observed and quantified. The observations took place twice daily between the hours of $9.00 \mathrm{am}$ and $12.00 \mathrm{a} . \mathrm{m}$. and between $4.00 \mathrm{pm}$ and $6.00 \mathrm{pm}$ and lasted for two days. The hours selected for the study fall within the peak periods for pedestrians and vehicular activity. It is also important to note that the observations were conducted under clear weather condition.

In the present study, the pace of pedestrians was determined in terms of observed walking tempo: regular (normal walking), running and stopping. Observations were made in the case of pedestrians' head movements towards four directions: (a) toward the traffic lights/warden (where available), (b) toward the left / right, (c) toward the other pedestrians, and (d) toward the ground formed (see Tom and Granié, 2011). These observed directions are important because the head movements to the right and left at crossing site offer the pedestrians the opportunity to make a safe judgment of the traffic situation before crossing the road.

\section{Statistical data analysis}

The data collected were coded and centralised electronically using Epidata software, an open source data entry software. The observed behaviours were ranked. A mark (1) was assigned for every safe and another (0) was assigned for unsafe behaviour. The data were analysed using descriptive statistics with IBM
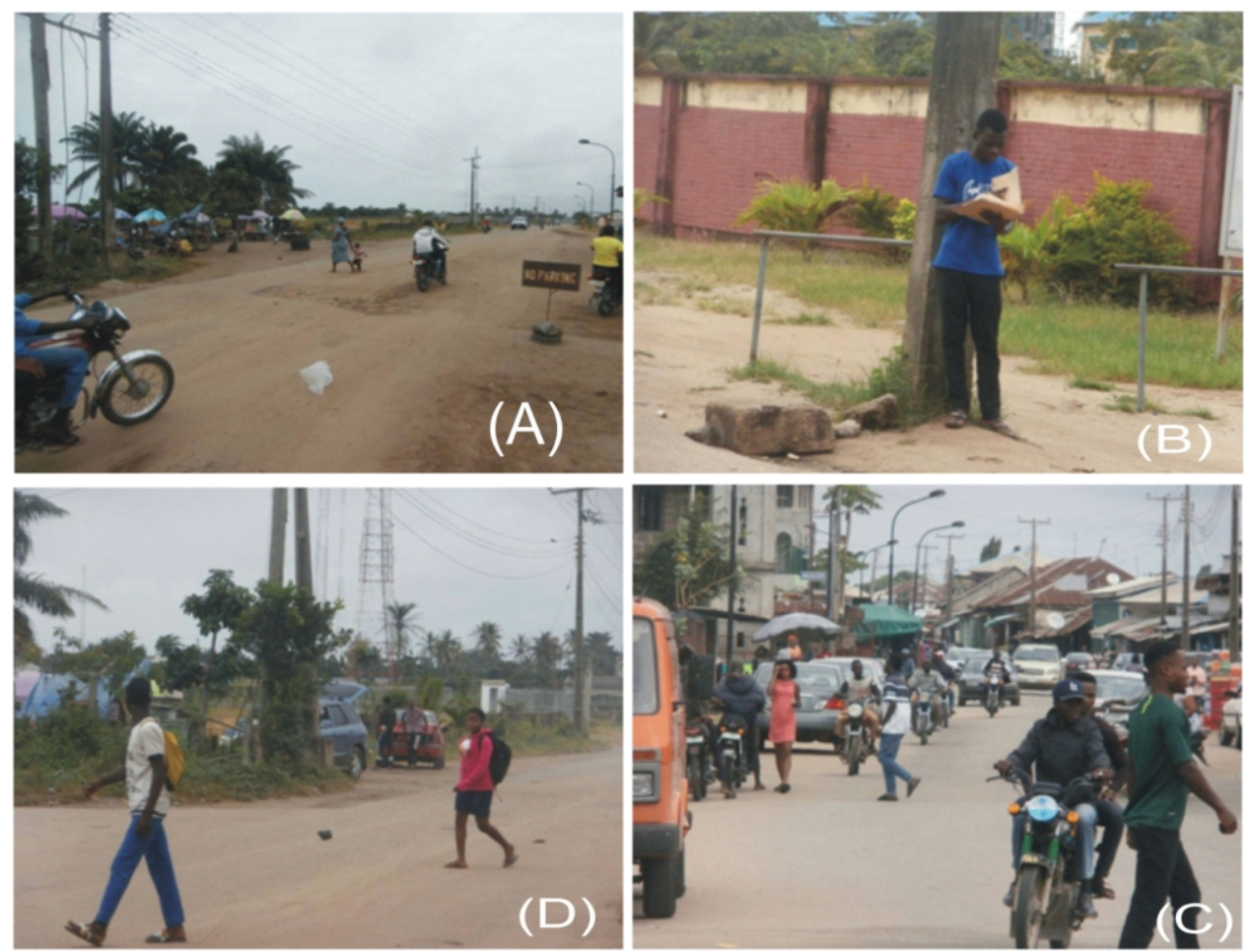

Photos 1A - 1D. Snapshots of some of the sampled streets 
Table 2. .Variables and associated codes used in logistic regression.

\begin{tabular}{lll}
\hline Variable & Case & Code \\
\hline Dependent & & \\
\hline & Unsafe & 0 \\
\cline { 2 - 3 } & Save & 1 \\
\hline Independent & & \\
\hline i. Sex & Female & 0 \\
\cline { 2 - 3 } & Male & 1 \\
\hline ii. Age & Youth & 1 \\
\cline { 2 - 3 } & Adult & 0 \\
\hline iii. Traffic Situation & No vehicular movement & 1 \\
\cline { 2 - 3 } & Vehicles moving on right or left sides & 1 \\
\cline { 2 - 3 } & Vehicles moving on both sides & 2 \\
\hline iv. Is pedestrian carrying / holding something & No & 0 \\
\cline { 2 - 3 } & Yes & 1 \\
\hline
\end{tabular}

Source: Authors Field Observation, 2017

SPSS software for Windows (Ver. 23). Statistically significant differences between pedestrian risky and safe crossing behaviour were based on Chi-square test, while Analysis of Variance (ANOVA) was used for spatial differences in safe and unsafe pedestrian behaviours among the study cities.

The data was further analysed using logistic regression model which is also used in similar studies by Zhang et al. (2014); Hassan and Al-Faleh (2013), MacLeod et al., (2012) and Rosenbloom (2009). Logistic regression was performed to ascertain the effects of gender, age, traffic situation and whether the observed pedestrian is holding or carrying something, on the likelihood that cumulative behaviour of the observed pedestrian will result into safe crossing behaviour (table 2).

\section{Results}

\section{Characteristics of pedestrians}

The total number of pedestrians observed was 1,214 consisting of male 730 $(60.1 \%)$ and female 484 (39.9\%). Adult pedestrians accounted for 58.2\%, while $41.8 \%$ of the samples are youths. The majority (57.6\%) of the sample pedestrians were observed crossing alone while $42.4 \%$ were selected for observation from groups of pedestrians crossing the road.

\section{Pedestrians safe and unsafe crossing behaviour}

The distribution of safe and unsafe crossing behaviours of pedestrians at each of the three street crossing stages is shown in table 3. The observed pedestrians exhibited varying proportions of safe and risky behaviour while approaching the Kerb, at the Kerb and while crossing the street (table 3).

\section{Head movements of pedestrians}

In terms of head movement to the left and right sides of the road, over $70 \%$ in each of the three study locations looked to either sides (left side V right side) of 
Table 3. Unsafe and safe head movement before, at the Kerb and while crossing.

\begin{tabular}{|c|c|c|c|c|c|c|c|c|}
\hline \multirow[t]{2}{*}{ Summary } & \multicolumn{2}{|c|}{ Ile-Ife (in \%) } & \multicolumn{2}{|c|}{ ljebu-Igbo (in \%) } & \multicolumn{2}{|c|}{ Badagry (in \%) } & \multicolumn{2}{|c|}{ Total (in \%) } \\
\hline & Unsafe & Safe & Unsafe & Safe & Unsafe & Safe & Unsafe & Safe \\
\hline Head Movement & Before $t$ & Kerb & 5m) & & & & & \\
\hline To left side & & & & & & & & \\
\hline No (0), Yes (1) & 9.2 & 90.8 & 27.1 & 72.9 & 27.4 & 72.6 & 20.9 & 79.1 \\
\hline To right side & & & & & & & & \\
\hline No (0), Yes (1) & 4 & 96 & 29.7 & 70.3 & 22.2 & 77.8 & 18.2 & 81.8 \\
\hline $\begin{array}{l}\text { To the Front } \\
\text { No (0), Yes (1) }\end{array}$ & 0 & 100 & 54.2 & 45.8 & 45.9 & 54.1 & 32.5 & 67.5 \\
\hline Head Movement & At the $\mathrm{Ke}$ & $(0.5 \mathrm{~m}$ & & & & & & \\
\hline $\begin{array}{l}\text { To left side } \\
\text { No (0), Yes (1) }\end{array}$ & 9.6 & 90.4 & 35.4 & 64.6 & 35.6 & 64.4 & 26.4 & 73.6 \\
\hline $\begin{array}{l}\text { To right side } \\
\text { No (0), Yes (1) }\end{array}$ & 4 & 96 & 36.5 & 63.5 & 31.1 & 68.9 & 23.3 & 76.7 \\
\hline $\begin{array}{l}\text { To the Front } \\
\text { No (0), Yes (1) }\end{array}$ & 0 & 100 & 56.2 & 43.8 & 43.7 & 56.3 & 32.4 & 67.6 \\
\hline Head Movement & While c & ssing & & & & & & \\
\hline $\begin{array}{l}\text { To left side } \\
\text { No (0), Yes (1) }\end{array}$ & 9.2 & 90.8 & 51.6 & 48.4 & 38.5 & 61.5 & 32.4 & 67.6 \\
\hline $\begin{array}{l}\text { To right side } \\
\text { No (0), Yes (1) }\end{array}$ & 4 & 96 & 96 & 44.8 & 34.1 & 65.9 & 30.2 & 69.8 \\
\hline $\begin{array}{l}\text { To the Front } \\
\text { No (0), Yes (1) }\end{array}$ & 0 & 100 & 100 & 56.8 & 37.8 & 62.2 & 26.3 & 73.7 \\
\hline
\end{tabular}

the road before crossing: Ile - Ife (90.8\% V 96\%), Ijebu - Igbo (72.9\% V 70.3\%) and Badagry (79.1\% V 81.8\%) before reaching the Kerb. Similar behaviour was observed for head movements to other directions before reaching the Kerb (Table 3). At the Kerb, significant safe head movement behaviours were observed among pedestriansin two of cities: Ile - Ife $(\chi 2=299.88, d=1, p=0.000)$ and Badagry $(\chi 2=13.89, \mathrm{~d}=1, \mathrm{p}=0.000)$, and during crossing: Ile - Ife $(\chi 2=306.64, d=1, p=0.000)$ and Ijebu - Igbo $(\chi 2=15.04, d=1, p=0.000)$.

There was no difference between male and female's safe or unsafe head movement behaviour before reaching the Kerb and while waiting at the Kerb for the entire sampled pedestrians. However, female exhibited safe head movement behaviour than male $(66.7 \%$ v 60, 7\%) while crossing the street. Figure 2 shows that adults' pedestrians exhibited a higher level of safe head movement than the youth at the three stages of observations - before reaching the Kerb (77.1\% v 68.2\%); at the Kerb (72.8\% v 62.3\%) and during crossing $(64.9 \% \mathrm{v} 60.6 \%)$. When the aggregate data was further analysed based on whether the observed pedestrian crossed the street alone or in a group, a significant level of safe head movement behaviour was observed among the individual samples and samples from the group of pedestrians.

\section{Tempo of pedestrians}

The tempo of pedestrians at the Kerb and during crossing was determined in terms of the pedestrian movement speed: normal walking, running or stopping. At the Kerb (0.5-0 metres), the majority of the observed pedestrians in the three target cities behaved in an unsafe manner by either running or walking continuously across the streets without pausing at the Kerb to assess the street's traffic conditions. 


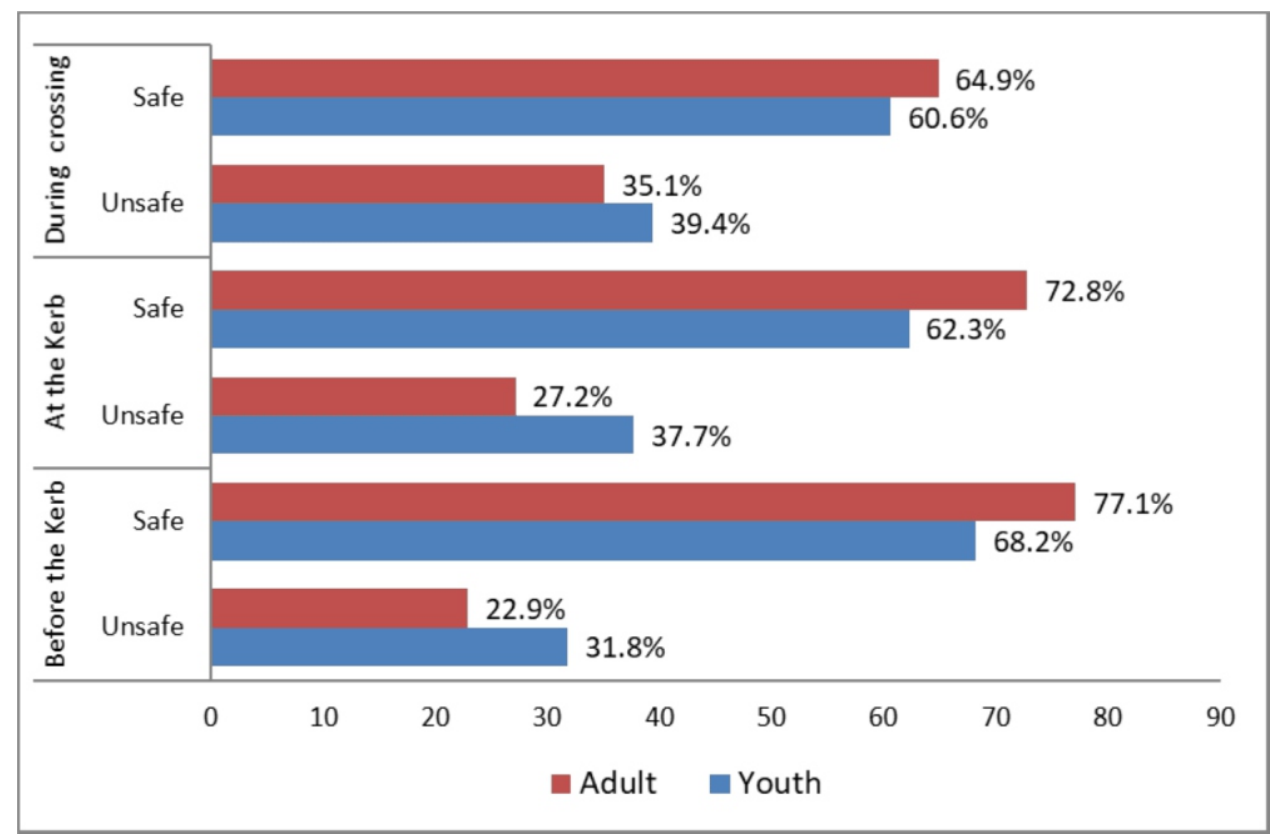

Figure 2. Pedestrians head movement by age

Table 4. Tempo during crossing by gender, age, and number of people crossing.

\begin{tabular}{llcccccc}
\hline Location & $\begin{array}{l}\text { Tempo while } \\
\text { crossing record }\end{array}$ & \multicolumn{2}{c}{ Sex (in \%) } & \multicolumn{3}{c}{ Age (in \%) } & \multicolumn{3}{c}{$\begin{array}{c}\text { Total number of } \\
\text { pedestrians (in \%) }\end{array}$} \\
\cline { 3 - 8 } & & Male & Female & Adult & Youth & Individual & Group \\
\hline Ile-Ife & Runs - Unsafe & 43.7 & 53.2 & 39.9 & 63.5 & 47.2 & 48.0 \\
\hline & Walking - Safe & 56.3 & 46.8 & 60.1 & 36.5 & 52.8 & 52.0 \\
\hline liebu-lgbo & Runs - Unsafe & 24.0 & 37.0 & 32.0 & 28.4 & 27.3 & 35.9 \\
\hline & Walking - Safe & 76.0 & 63.0 & 68.0 & 71.6 & 72.7 & 64.1 \\
\hline Badagry & Runs - Unsafe & 34.8 & 41.9 & 30.7 & 45.0 & 33.8 & 40.0 \\
\hline & Walking - Safe & 65.2 & 58.1 & 69.3 & 55.0 & 66.2 & 60.0 \\
\hline Total & Runs - Unsafe & 34.9 & 44.0 & 34.8 & 43.8 & 36.2 & 41.7 \\
\hline & Walking - Safe & 65.1 & 56.0 & 65.2 & 56.2 & 63.8 & 58.3 \\
\hline
\end{tabular}

Source: Field survey, 2017

There were gender differences in the unsafe tempo of male $(65.2 \%)$ and female $(54.5 \%)$ pedestrians at the Kerb. Adult pedestrians (62.7\%) exhibited unsafe behaviour in terms of not stopping at the Kerb before proceeding to cross than the youths (58.6\%) at the Kerb. The observed tempo of pedestrians at the Kerb was about the same in terms of risky crossing behaviour. About $62.5 \%$ of individuals and $59.2 \%$ of pedestrian observed within groups of people either run or continue walking across the street without stopping at the Kerb.

During the crossing of the street, safe tempo behaviour was observed among male $(65.1 \%)$ than female $(56.0 \%)$ pedestrians (Table 4 ). Adult pedestrians $(62.7 \%)$ exhibited significant safe behaviour in terms of tempo compared to youths (58.6\%). A higher percentage of individuals crossing (63.8\%) as compared to observed pedestrians in a group (58.3\%) crossed in a safe manner (see Table 4). More male than female in the three cities studied behaved in a safe way. In the same manner, adult more than youth exhibited safe behaviour in terms of walking pace (Table 4). 


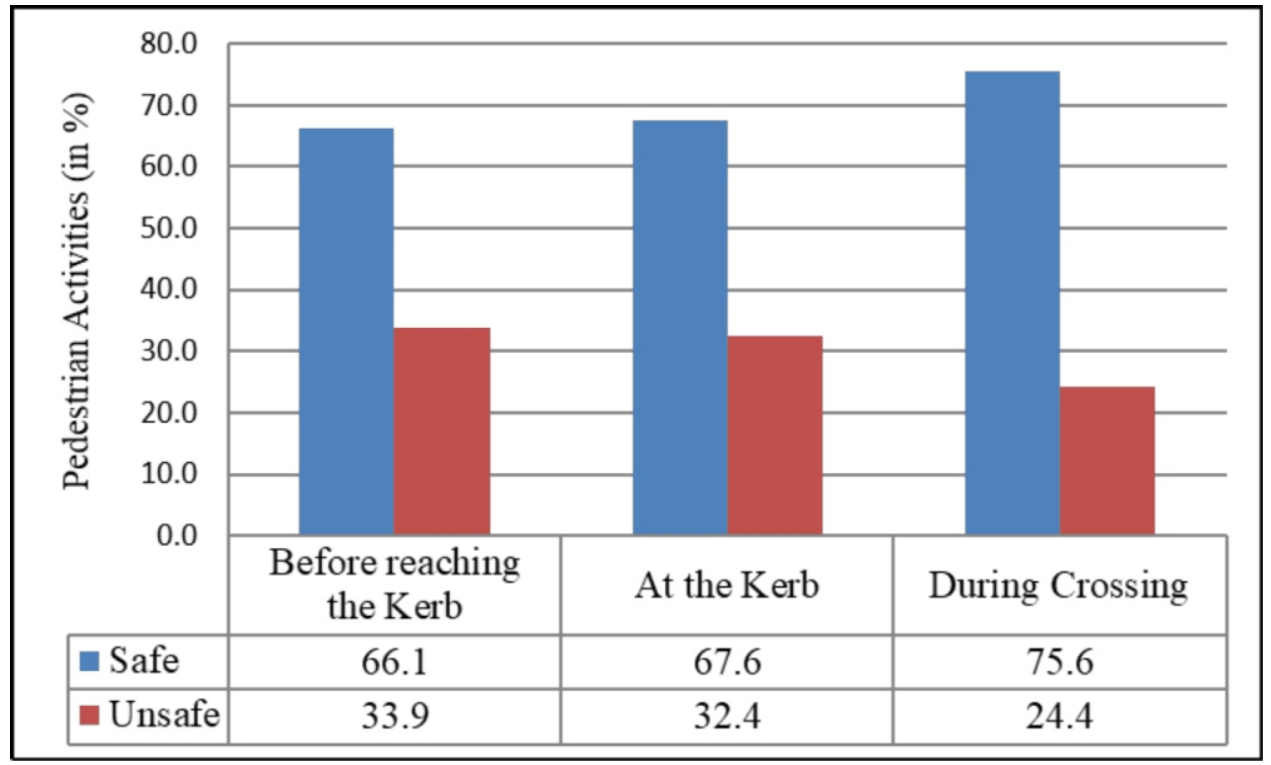

Figure 3. Pedestrians' involvement in distractive activities

Pedestrians' involvement in distractive activities

Distractive activities such as talking, having a conversation on mobile phones or having headphone on, were observed and classified as unsafe behaviour. Those who are not involved in any of these activities were classified as exhibiting crossing safe behaviour. Majority of the pedestrians observed were not involved in any activities that negate safety principles at the three stages of observations in the sampled cities. The proportions of safe behaviour in terms of noninvolvement in distractive activities are $66.1 \%$ before reaching the Kerb, at the Kerb (67.6\%) and 75.6\% during street crossing (Figure 3).

\section{Standing Position before crossing}

Most roads in the sample cities lack sidewalk; hence, the road edges mostly used by pedestrians were considered as sidewalks in this study. A high proportion of the observed pedestrians in the studied cities stood on the sidewalk $(63.5 \%)$, an indication of safe behaviour, as compared to $36.5 \%$ of the observed pedestrians that stood on the road surface before crossing. This result differs according to cities. There was no significant difference in safe standing position before crossing in the whole sample in terms of gender: male $(64.4 \%)$ vs. female $(62.2 \%)$; age: adults $(63.2 \%)$ vs. youths $(63.9 \%)$ and number of pedestrians crossing - individuals $(65.2 \%)$ vs. pedestrians that were crossing in group $(61.5 \%)$.

\section{Traffic situation during a crossing}

Traffic situation plays a crucial role in determining the safety of pedestrians during a crossing. Vehicular movement on both sides of the road during crossing will increase accident risk to the pedestrian. The traffic situation during 
Table 5. Traffic situation during a crossing.

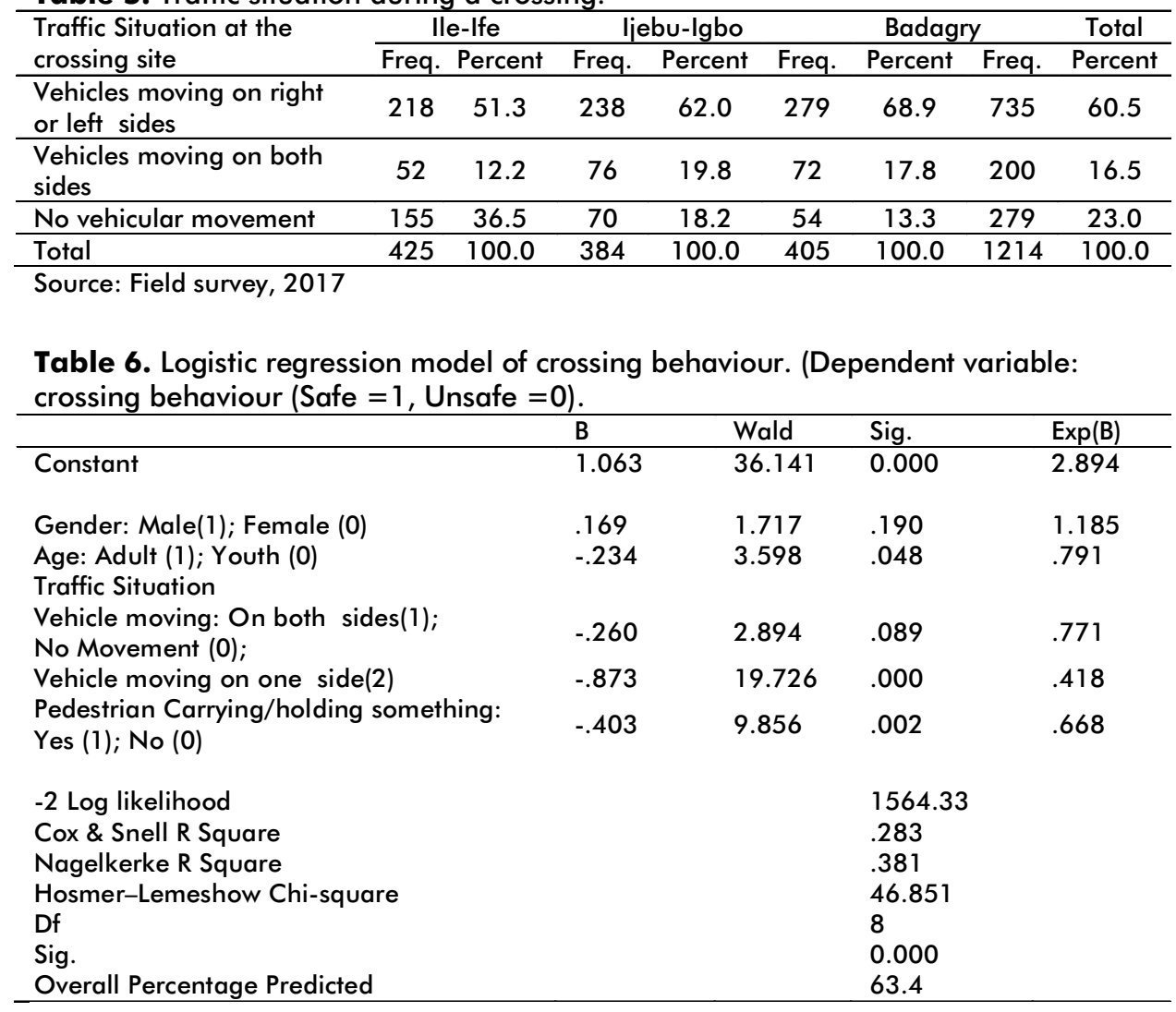

crossing is quantified in this study with respect to three traffic situations: vehicles moving on right or left sides; vehicles moving on both sides, and no vehicular movement. Majority of the pedestrians (60.5\%) commenced crossing when the vehicular movement is from one side of the road (Table 5). About $23 \%$ and $16.6 \%$ of the observed population crossed the road when there were no vehicular movement and when vehicles moved on both side of the road, respectively.

\section{Logistic regression analyses}

The result of the logistic regression performed on the data collected as evident in table 6 showed that the model was statistically significant, $\chi^{2}(8)=46.851, p<$ .000 . The model explained $38 \%$ (Nagelkerke $\mathrm{R} 2=0.38$ ) of the variance in safe crossing behaviour and correctly classified $63.4 \%$ of cases. The Wald criterion demonstrated that crossing when vehicles are moving on both sides of the road; crossing while carrying/holding something, and crossing as an adult pedestrian made a significant contribution to the prediction $(p<.05)$. $\operatorname{EXP}(B)$ value indicates that the odds of crossing in a safe manner is 1.18 times higher for males as opposed to females. Table 6 showed that adult pedestrians are 0.79 times more likely to exhibit safe crossing than younger pedestrians. It also revealed that pedestrians are 0.42 times more likely to exhibit safe crossing 
when vehicles are moving on one side of the road than when there are no vehicular movement. In addition, pedestrians are 0.67 times more likely to exhibit safe crossing when carrying/holding something than when they are not carrying/holding something.

\section{Discussion}

The aim of the study was to examine pedestrians' street crossing behaviour in three cities in South Western Nigeria. Findings showed that significant similarities and differences in crossing behaviour in among sample cities. An important finding of the study is that majority of the pedestrians' in these cities looked to left and right and sides for moving vehicles especially at the Kerb before crossing and during crossing the street. This confirms the findings of a study by Seipone et al. (2013) which reported that the majority of pedestrians check both sides of the road before crossing. This behaviour is expected as most of the streets in the study areas lack pedestrian walkways and zebra crossing sites/ traffic control post (Odeleye, 2002).

Furthermore, the results indicated that male and female behaved similarly in their head movement while assessing traffic situation before crossing. During crossing high proportions of female than male exhibited safe head movement behaviour. While, Pedestrian crossing alone showed a significant level of safety awareness in terms of head movements before reaching the Kerb and at the Kerb than those observed crossing in a group of people. In addition, the results indicate that majority of pedestrians in Ijebu-Igbo and Badagry behaved in an unsafe manner in terms of their tempo at the Kerb. Male more than female pedestrians also differ in pace as they either run or walk continuously from the Kerb to the streets without waiting at the Kerb to check for coming vehicles.

During the crossing, gender and age differences in pedestrians' pace were also found in all the cities. Higher proportions of male than female and more adults than youths crossed the streets walking. This finding differs from the findings of Rosenbloom et al. (2008) that shows that running is a common behaviour at crosswalks and that of Zhuang and Wu (2011) which revealed that pedestrians examined traffic situations and quickly or run across the roadway to their destinations when satisfied that the roadway is safer. A high level of diagonal street crossing behaviour (unsafe crossing behaviour) was revealed by our study. This also varied based on gender, age and group membership. More male pedestrians crossed the road diagonally (unsafe crossing) than female. Pedestrians in group members often crossed streets diagonally more than an individual. This finding corroborates Rosenbloom's (2009) findings in the study of individual and group behaviour at a red light found that, compared to female pedestrians, male pedestrians more often crossed the road at the red light (unsafe crossing).

Contrary to general beliefs that pedestrians take an unnecessary risk at crossing sites, this study shows that majority of pedestrians are compliant with traffic rules in the study areas by not engaging in distractive activities at the three stages of observations. This finding deviates from several findings that established high participation in distractive activities such as talking, using a 
mobile phone or listening to personal music device during street crossing (Pesic et al., 2016; Nasar and Troyer, 2013; Neider et al., 2011; Nasaret al., 2008).

\section{Implications and Conclusion}

The importance of this research is that it described in detail the pedestrian behaviour while approaching the Kerb, at the Kerb and during street crossing in three medium-sized cities in South Western Nigeria. The findings call for attention, particularly at the local, state and national levels.At the national level, the government is encouraged to designate zebra crossing sites on main roads where necessary and install Zebra crossing signs on potential crossing sites in major cities in the country. These are meant to reduce the rate of pedestrians' accidents associated streets crossing. In addition, an effective education programme on the use of pedestrian facilities should be put in place. Raising the awareness level of the relevance of common pedestrian facilities, such as a pedestrian walkway, zebra crossing, pedestrians bridges and traffic officers in the country will contribute immensely towards pedestrians' safety in the country.

The study has brought to light the salient aspects of pedestrians crossing behaviours inherent in medium cities in Nigeria through the use of direct observation method. The study methodology can be improved and applied to study the different aspects of pedestrians crossing behaviour such as waiting time at crossing sites, pedestrians-drivers' interactions and acceptable safe gap especially at non-zebra and at unsignalised crossing sections in other medium sized cities and major cities in the country.

\section{References}

Aaladelusi, T.O., Akinmoladun, I.V, Olusanya, O.O, Akadiri, O.A. and Fasola, A.O. (2014), "Evaluation of pedestrian road traffic maxillofacial injuries in a Nigerian tertiary hospital", Afr J Med Med Sci, no.4, p. 353-9.

Bungum, T.J., Day, C., and Henry, J. (2005), "The association of distraction and caution displayed by pedestrians at a lighted crosswalk", Journal of Community Health, vol. 30, no. 4, p. 269-279.

Damsere-Derry, J., Ebel, B.E., Mock, C.N., Afukaar, F. and Donkor, P. (2010), "Pedestrians' injury patterns in Ghana", Accident Analysis E Prevention, vol. 42, no. 4, p. 1080-1088.

Diaz, E.M. (2002), "Theory of planned behaviour and pedestrians' intentions to violate traffic regulations", Transportation Research Part F: Traffic Psychology and Behaviour, vol. 5, p. 169-175.

Hassan, H.M. and Al-Faleh, H. (2013), "Exploring the risk factors associated with the size and severity of roadway crashes in Riyadh", Journal of Safety Research, vol. 47, no. 2013, p. 67-74.

Hatfield, J. and Murphy, S. (2007), "The effects of mobile phone use on pedestrian crossing behaviour at signalized and unsignalised intersections", Accident Analysis and Prevention, vol. 39, p. 197-205.

Huth, V., Sanchez, Y. and Brusque, C. (2014), "Drivers' phone use at red traffic 
lights: A roadside observation study comparing calls and visual-manual interactions", Accident Analysis and Prevention, vol. 74, p. 42-48.

MacLeod, K.E., Griswold, J.B., Arnold, L.S. and Ragland, D.R. (2012), "Factors associated with hit-and-run pedestrian fatalities and driver identification", Accident Analysis and Prevention, vol. 45, p. 366-372.

Nasar, J. and Troyer, D. (2013), "Pedestrian injuries due to mobile phone use in public places", Accident Analysis and Prevention, vol. 57, p. 91-95.

Nasar, J., Hecht, P. and Werner, R. (2008), "Mobile telephones, distracted attention, and pedestrian safety", Accident Analysis and Prevention, vol. 40, p. 69-75.

National Population Commission (NPC) (2006), Official Gazatte (FGP 71/52007/2,500/OL24): Legal Notice on Publication of the Details of the Breakdown of the National and State Provisional Totals 2006 Census.

Neider, M.B., McCarley, J.S., Crowell, J.A., Kaczmarski, H. and Kramer, A.F. (2010), "Pedestrians, vehicles, and cell phones", Accident Analysis and Prevention, vol. 42, p. 589-594.

Odeleye, J.A. (2002), "Zebra crossing and pedestrian safety management in road traffic environment in Metropolitan Lagos, Nigeria", $15^{\text {th }}$ ICTCT Workshop on Speed management strategies and implementation planning, evaluation, behavioural, legal and institutional issues, Brno, Czech Republic, October 23- 25.

Ogendim J., Odero, W., Mitullah, W. and Khayesi, M. (2013), "Pattern of pedestrian injuries in the city of Nairobi: implications for urban safety planning", Journal of Urban Health: Bulletin of the New York Academy of Medicine.

Olawole, M.O. (2008), "Accessibility characteristics of rural population in Badagry Local Government Area of Lagos State", M.Sc Thesis, Department of Geography, Obafemi Awolowo University, Ile-Ife, Nigeria.

Olawole, M.O. (2017), "Gender aspects of street crossing behaviour among undergraduates: An observational study", Ghana Journal of Geography, vol. 9, no. 3, p. 1-22.

Parker, D., Manstead, A.S.R., Stradling, S.G., Reason, J.T. and Baxter, J.S. (1992), "Intention to commit driving violations: An application of the theory of planned behavior", Journal of Applied Psychology, vol. 77, no. 1, p. 94-101.

Pešić, D., Antić, B., Glavić, D. and Milenkovic, M. (2016), "The effects of mobile phone use on pedestrian crossing behaviour at unsignalized intersections Models for predicting unsafe pedestrians' behaviour", Safety Science, vol. 82, p. $1-8$.

Poo, F.M., Ledesma, R.D. and Trujillo, R. (2018), "Pedestrian crossing behavior, an observational study in the city of Ushuaia, Argentina", Traffic Injury Prevention, vol. 19, no. 3, p. 305-310.

Rosenbloom, T., Nemrodov, D. and Barkan, H. (2004, "For heaven's sake follow the rules: pedestrians' behavior in an ultra-orthodox and a non-orthodox city", Transportation Research Part F: Traffic Psychology and Behaviour, vol. 7, no. 6, p. 395-404.

Rosenbloom, T., Ben-Eliyahu, A. and Nemrodov, D. (2008), "Children's crossing behaviour with an accompanying adult", Safety Science, vol. 46, p. 1248-1254.

Rosenbloom, T. (2009), "Crossing at a red light: Behaviour of Individuals and groups", Transportation Research Part F: Traffic Psychology and Behaviour, vol. 12, p. 389 - 394. 
Rosenbloom, T. (2011), "Traffic light compliance by civilians, soldiers and military officers", Accident Analysis and Prevention, vol. 43, p. 2010- 2014.

Seipone B.M., Mphele, S.B.M., Selemogwe, M.M., Kote, M. and Balogun, S.K. (2013), "Who Owns the Road? Exploring Driver and Pedestrian Behaviour at Zebra/Pedestrian Crossings in Gaborone, Botswana", British Journal of Arts and Social Science, vol. 13, no. 1, p. 121-130.

Sisiopiku, V.P. and Akin, D. (2003), "Pedestrian behaviours at and perceptions towardsvarious pedestrian facilities: an examination based on observation and survey data", Transportation Research Part F: Traffic Psychology and Behaviour, vol. 6, p. 249-274.

Solagberu, B.A., Balogun, R.A., Mustafa, I.A., Ibrahim, N.A., Oludara, M.A. Ajani, A.O., Idowu, O.E. and Osuoji, R.I. (2015), "Pedestrian Injuries in the Most Densely Populated City in Nigeria - An Epidemic calling for Control", Traffic Injury Prevention, vol. 16, no. 2, p. 184-189.

Tiwari, G., Bangdiwala, S. and Saraswat, A. (2007), "Survival Analysis: Pedestrian Risk Exposure at Signalized Intersections", Transportation Research Record: Journal of the Transportation Research Board, vol. 10, p. 77-89.

Tom, A. and Granié, M.-A. (2011), "Gender differences in pedestrian rule compliance and visual search at signalized and unsignalized crossroads", Accident Analysis E Prevention, vol. 43, no. 5, p. 1794-1801.

WHO, (2015), World Health Organization - Global Status report on road safety 2015, p.340, viewed 12 March 2017, https://goo.gl/YXHdWZ.

Yagil, D. (2000), "Beliefs, motives and situational factors related to pedestrians' self-reported behaviour at signal-controlled crossings", Transportation Research Part F: Traffic Psychology and Behaviour, vol. 3, no. 1, p. 1-13.

Zhang, G., Li, G., Cai, T., Bishai, D.M., Wu, C. and Chan Z. (2014), "Factors contributing to hit-and-run crashes in China", Transportation Research Part F: Traffic Psychology and Behaviour, vol. 23, p. 113-124.

Zhou, R., Horrey, W. J. and Yu, R. (2009), "The effect of conformity tendency on pedestrians' road-crossing intentions in China: An application of the theory of planned behavior", Accident Analysis and Prevention, vol. 41, no. 3, p. 491-97.

Zhuang, X. and Wu, C. (2011), "Pedestrians' crossing behaviours and safety at unmarked roadway in China", Accident Analysis and Prevention, vol. 43, no. 6, p. $1927-1936$. 